\title{
Preface: Special Issue on Homotopy Type Theory and Univalent Foundations
}

\author{
Peter Lefanu Lumsdaine ${ }^{1} \cdot$ Nicolas Tabareau $^{2}$ (B)
}

Received: 11 October 2018 / Accepted: 21 October 2018 / Published online: 30 October 2018

(c) Springer Nature B.V. 2018

This preface introduces the first special issue out of a series of workshops on Homotopy Type Theory and Univalent Foundations. This recent area of research finds its roots in the seminal work of Martin Hofmann and Thomas Streicher on the structure of Martin-Löf identity types [1]. But the main research program has been foreseen by Vladimir Voevodsky, who, from its initial motivation of formalizing his results in homotopy theory, has initiated what is now called the univalent foundations program [2]. Borrowing ideas from homotopy theory, the goal of the univalent foundations program is to leverage dependent Type Theory to a formal framework that could replace Set Theory for the foundations of mathematics.

This special issue gathers research contributions of some of the most prominent researchers of the field that we briefly outline below.

The univalence axiom in cubical sets. Authors: Marc Bezem, Thierry Coquand and Simon Huber.

This paper shows that the central axiom of the univalent foundations holds in a model of Type Theory based on (Kan) cubical sets. The univalence axiom has originally been justified by Vladimir Voevodsky using the simplicial sets model, but the cubical sets model is the first model of a univalent type theory which can be expressed in a constructive setting and thus is amenable to a concrete implementation.

\section{Canonicity for Cubical Type Theory. Author: Simon Huber}

Cubical type theory is a univalent type theory reflecting the structure of the cubical sets model where there is a direct access to the manipulation of n-dimensional cubes. In this type theory, the univalence axiom is provable. This paper shows that cubical type theory satisfies a canonicity property which implies in particular that any natural number in a context build from only name variables is judgmentally equal to a numeral. This result shows that cubical type theory is a convenient theory to be implemented and to compute with.

Guarded Cubical Type Theory. Authors: Lars Birkedal, Aleš Bizjak, Ranald Clouston, Hans Bugge Grathwohl, Bas Spitters and Andrea Vezzosi.

To Martin Hofmann and Vladimir Voevodsky.

Nicolas Tabareau

nicolas.tabareau@inria.fr

1 Stockholm University, Stockholm, Sweden

2 Inria, Nantes, France 
This papers proposes to extend Guarded Type Theory, a type theory with guarded recursive types, to a cubical setting, in order to provide better reasoning on equality proofs. The resulting theory provides a computational interpretation of extensionality for guarded recursive types.

The James construction and $\pi_{4}\left(\mathbb{S}^{3}\right)$ in homotopy type theory. Author: Guillaume Brunerie.

This paper is part of a recent area of research emerging from Homotopy Type Theory and its facility to formalize homotopy theoretic constructions directly in type theory, what is called synthetic homotopy theory. More precisely, this paper presents the formalization in Agda of the James construction and use it to prove that the fourth homotopy group of the 3 -dimensional sphere is of the form $\mathbb{Z} / n \mathbb{Z}$, which is a standard result in homotopy theory.

From signatures to monads in UniMath. Authors: Benedikt Ahrens, Ralph Matthes, Anders Mörtberg.

This work is about formalizing the construction of initial algebras in UniMath, which is a core type theory based on the univalent foundations, making use of as few type constructors as possible, originally initiated by Vladimir Voevodsky. The idea of UniMath is to keep the kernel of theory as small as possible, in order to provide a minimal foundational theory. This work about initial algebras is thus a way to add some kind of inductive types in the theory of UniMath and there associated induction principles.

\section{References}

1. Hofmann, M., Streicher, T.: The groupoid interpretation of type theory. Twenty-Five Years of Constructive Type Theory (Venice. 1995), Oxford Logic Guides, vol. 36, pp. 83-111. Oxford University Press, New York (1998)

2. The Univalent Foundations Program.: Homotopy Type Theory: Univalent Foundations of Mathematics. Institute for Advanced Study. http://homotopytypetheory.org/book (2013) 